\title{
Nutrientes afetando as mudas de alecrim-pimenta (Lippia sidoides Cham.) e seus artrópodes
}

\author{
SILVA, F.W.S. ; LEITE, G.L.D. ${ }^{*}$; GUANABENS, R.E.M. ${ }^{1}$; MARTINS, E.R. ${ }^{1}$; MATIOLI, A.L ${ }^{2}$; FERNANDES, L.A. ${ }^{1}$ \\ 'UFMG, Setor de Fitotecnia, Núcleo de Ciências Agrárias, Av. Osmane Barbosa, s/n, B. JK, Cx. Postal 135, CEP: \\ 39404-006, Montes Claros, MG. *e-mail: gldleite@ufmg.br ${ }^{2}$ Instituto Biológico, Laboratorio de Entomologia \\ Econômica, Rodovia Heitor Penteao, km 3, Jardim das Palmeiras, Cx. Postal 70, CEP: 13012-970, Campinas-SP. \\ email:almatioli@biologico.sp.gov.br
}

RESUMO: O objetivo deste trabalho foi avaliar o efeito da omissão de nutrientes nas mudas de Lippia sidoides Cham. (Verbenaceae) e seu possível efeito sobre os seus artrópodes, sendo os tratamentos: 1) testemunha; 2) completo 1 adubado com N, P, K, S, B, Cu, e Zn + calagem (C1); 3) completo 2: C1 sem calagem + Ca e Mg como sulfato (C2); 4) C1 sem calagem;5) C1 sem N; 6) $C 1$ sem $P$; 7) $C 1$ sem $K$; 8) $C 1$ sem $S$; 9) $C 1$ sem $B$; 10) $C 1$ sem $C u$; 11) $C 1$ sem $Z n$; 12) $C 2$ sem $\mathrm{Ca}$ e 13) $\mathrm{C} 2 \mathrm{sem} \mathrm{Mg}$. O delineamento foi inteiramente casualizado com quatro repetições. O Tetranychus sp. (Acari: Tetranychidae) atacou mais os tratamentos 3, 6, 9 e 13 e os maiores danos nos tratamentos 6 e 13. A maior população de Aphis gossypii Glover (Homoptera: Aphididae) foi encontrada nos tratamentos 5, 6, 7, 8, 11 e 13, colonizando preferencialmente o tratamento 4. O Phenacoccus sp. (Hemiptera: Pseudococcidae) foi encontrado em maior número nos tratamentos 4 e 9 e Insignorthezia insignis (Browne) (Hemiptera: Ortheziidae) em 13. Foram mais notados adultos de Bemisa tabaci (Genn.) (Hemiptera: Aleyrodidae) nos tratamentos 5, 7, 8 e 13 e ninfas nos três últimos tratamentos. Em geral, os tratamentos 1,5 e 10 são os menos atacados por artrópodes. Dirigir a pulverização, quando necessário, sempre para a face inferior da folha.

Palavras-chaves: plantas medicinais, adubação, insetos, ácaros

\begin{abstract}
Nutrients affecting "alecrim-pimenta" (Lippia sidoides Cham.) seedlings and their arthropods. The aim of this work was to evaluate nutrient omission effect on Lippia sidoides Cham. (Verbenacea) seedlings, as well as its possible effect on their arthropods. Treatments were: 1) control; 2) complete 1: fertilized with $\mathrm{N}, \mathrm{P}, \mathrm{K}, \mathrm{S}, \mathrm{B}, \mathrm{Cu}$, and $\mathrm{Zn}+$ lime (C1); 3) complete 2: $\mathrm{C} 1$ without lime $+\mathrm{Ca}$ and $\mathrm{Mg}$ as sulphate $(\mathrm{C} 2)$; 4) $\mathrm{C} 1$ without lime; 5) $\mathrm{C} 1$ without $\mathrm{N}$; 6) $\mathrm{C} 1$ without $P$; 7) $C 1$ without $K$; 8) $C 1$ without $S$; 9) $C 1$ without $B$; 10) $C 1$ without $C u$; 11) $C 1$ without $Z n$; 12) $\mathrm{C} 2$ without $\mathrm{Ca}$; and 13) $\mathrm{C} 2$ without $\mathrm{Mg}$. The experimental design was completely randomized, with four replicates. Tetranychus sp. (Acari: Tetranychidae) predominantly attacked treatments 3 , 6,9 and 13 , and the greatest damages were detected in treatments 6 and 13. The largest population of Aphis gossypii Glover (Homoptera: Aphididae) was observed in treatments 5, 6, 7, 8, 11 and 13, preferentially colonizing treatment 4 . Phenacoccus sp. (Hemiptera: Pseudococcidae) was predominant in treatments 4 and 9, and Insignorthezia insignis (Browne) (Hemiptera: Ortheziidae), in treatment 13. Bemisia tabaci (Genn.) (Hemiptera: Aleyrodidae) adults were mostly detected in treatments 5, 7, 8 and 13, whereas nymphs, in the last three treatments. In general, treatments 1,5 and 10 are the least attacked by arthropods. When needed, pulverization must always be directed to the leaf lower surface.
\end{abstract}

Key words: medicinal plants, fertilization, insects, mites

Recebido para publicação em 14/08/2007

Aceito para publicação em 05/08/2008

Rev. Bras. PI. Med., Botucatu, v.11, n.1, p.18-23, 2009. 


\section{INTRODUÇÃO}

O alecrim-pimenta (Lippia sidoides Cham.) (Verbenacea) é originária do Brasil, sendo propagada por estaquia ou alporquia. Seus principais constituintes químicos são os óleos essenciais, contendo mais de $60 \%$ de timol ou uma mistura de timol e cavacrol, possuindo ação antimicrobiana contra infecções da garganta, cárie dentária, impingens, acne, pano-branco, aftas, escabiose, caspa, maus odores dos pés e axilas, sarnainfecciosa e pé-de-atleta, antiespasmódico e estomáquico (Martins et al., 1994; Lorenzi \& Matos, 2002).

Os compostos tectol e lipsidoquinona exibem atividade significante contra células leucêmicas humanas (Costa et al., 2001; Carvalho et al., 2003; Girão et al., 2003). Nos cachorros, o óleo essencial dessa planta reduz a gengivite marginal causada por bactérias; e apresenta ação larvicida contra o mosquito Aedes aegypti L. (Diptera: Culicidae) (Carvalho et al., 2003; Girão et al., 2003).

O cultivo de plantas medicinais torna-se uma alternativa interessante e rentável no que tange a agricultura brasileira (Simões et al., 2000) e, portanto, é necessário se conhecer as exigências nutricionais da planta para cultivos comerciais. A nutrição é um dos fatores que podem interferir na composição química das plantas medicinais, interferindo diretamente na produção de biomassa e na quantidade de princípios ativos (Mapeli et al., 2005). Venturin et al. (1999) verificaram que a omissão de nutrientes limitou o crescimento de angico-amarelo (Peltophorum dubium Spreng.) (Caesalpinieae) e afetou também a absorção de enxofre pelas plantas. O desenvolvimento inicial de mudas de teca (Tectona grandis L.F.) (Verbenaceae) é afetado pela omissão de todos os macronutrientes, sendo que os danos mais intensos e imediatos são observados na ausência de $\mathrm{N}$ e $\mathrm{Ca}$, causando inclusive o apodrecimento das raízes secundárias e paralisação na emissão de novas raízes (Barroso et al., 2005).

As espécies medicinais normalmente apresentam resistência ao ataque de doenças e pragas, mas, por algum desequilíbrio, este pode ocorrer em níveis prejudiciais. Sendo assim, a nutrição, associada ao fator alimento é muito importante sobre a distribuição (no dossel e no período de cultivo) e abundância dos insetos, influenciando direta ou indiretamente a população destes, nos processos biológicos, morfológicos e de comportamento (Gallo et al., 2002).

Desta forma, objetivo do trabalho foi avaliar o efeito da omissão de macro (N, P, K, Ca e Mg) e micronutrientes (S, B, Cu e Zn) sobre as mudas de alecrim-pimenta e o possível efeito sobre as densidades dos seus artrópodes, bem como a distribuição destes no dossel e no período de cultivo.

\section{MATERIAL E MÉTODO}

O experimento foi realizado de agosto a dezembro de 2006, em condições de casa-devegetação do Núcleo de Ciências Agrárias (NCA) da Universidade Federal de Minas Gerais, em Montes Claros-MG. Utilizaram-se amostras de solo coletadas da camada de $0-20 \mathrm{~cm}$ de profundidade, de um Latossolo Vermelho Amarelo distrófico e álico (Embrapa, 1999), sob vegetação de cerrado, do município de Montes Claros, MG, com os seguintes atributos, determinados conforme Embrapa (1997): $\mathrm{pH}$ em água 4,1; $\mathrm{P}, 1 \mathrm{mg} \mathrm{dm}^{-3} ; \mathrm{Ca}, 2 \mathrm{mmol}_{\mathrm{c}} \mathrm{dm}^{-3} ; \mathrm{Mg}$, $1 \mathrm{mmol}_{\mathrm{c}} \mathrm{dm}^{-3} ; \mathrm{K}, 0,5 \mathrm{mmol}_{\mathrm{c}} \mathrm{dm}^{-3} ; \mathrm{Al}, 20 \mathrm{mmol}_{\mathrm{c}} \mathrm{dm}^{-3}$; $\mathrm{H}+\mathrm{Al}, 100 \mathrm{mmol}_{\mathrm{c}} \mathrm{dm}^{-3} ; \mathrm{Zn}, 0,3 \mathrm{mg} \mathrm{dm}^{-3} ; \mathrm{Mn}, 2 \mathrm{mg}$ $\mathrm{dm}^{-3}$; Fe, $6 \mathrm{mg} \mathrm{dm}^{-3}$; Cu, 0,1 mg dm${ }^{-3} ; \mathrm{B}, 0,1 \mathrm{mg} \mathrm{dm}^{-3}$; matéria orgânica, $20 \mathrm{~g} \mathrm{~kg}^{-1}$; areia, $500 \mathrm{~g} \mathrm{~kg}^{-1}$; silte, 80 $\mathrm{g} \mathrm{kg}^{-1} \mathrm{e}$ argila, $420 \mathrm{~g} \mathrm{~kg}^{-1}$.

$O$ delineamento experimental utilizado foi inteiramente casualizado com quatro repetições, sendo que cada parcela constituiu-se de duas plantas/ vaso ( $3 \mathrm{dm}^{3}$ de capacidade). As mudas foram feitas por estaquia em leito de enraizamento, sendo o substrato areia lavada e umidade de $75 \%$ a $85 \%$, e um mês depois, replantadas em agosto nos vasos, com as avaliações começando no mês subseqüente.

O experimento consistiu de 13 tratamentos baseados na técnica do diagnóstico por subtração (Fernandes et al., 2005): 1) testemunha; 2) completo 1: adubado com N, P, K, S, B, Cu, e Zn + calagem (C1);3) completo 2: completo 1 sem calagem + Ca e Mg como sulfato; 4) completo 1 sem calagem; 5) completo 1 sem nitrogênio; 6) completo 1 sem fósforo; 7) completo $1 \mathrm{sem}$ potássio; 8) completo $1 \mathrm{sem}$ enxofre; 9) completo 1 sem boro; 10) completo $1 \mathrm{sem}$ cobre; 11) completo 1 sem zinco; 12) completo $2 \mathrm{sem}$ cálcio e 13) completo 2 sem magnésio.

Nos tratamentos que receberam calcário, as doses foram baseadas em curvas de incubação, visando elevar a saturação por bases (V) a 70\% (Ribeiro et al., 1999). Usou-se o calcário dolomítico calcinado e micropulverizado, com $36 \%$ de $\mathrm{CaO}, 14 \%$ de $\mathrm{MgO}$ (Poder Relativo de Neutralização Total igual a $100 \%$ ), $0,02 \%$ de $\mathrm{Zn}, 0,01 \%$ de $\mathrm{B}, 0,03 \%$ de $\mathrm{Fe}$, $0,03 \%$ de $\mathrm{Mn}$ e $0,00 \%$ de $\mathrm{Cu}$. Foi aplicado por $\mathrm{dm}^{3}$ de solo, $100 \mathrm{mg}$ de $\mathrm{N}, 300 \mathrm{mg}$ de $\mathrm{P}, 100 \mathrm{mg}$ de K, $200 \mathrm{mg}$ de $\mathrm{Ca}, 60 \mathrm{mg}$ de $\mathrm{Mg}, 40 \mathrm{mg}$ de S, 0,5 mg de $\mathrm{B}, 1,5 \mathrm{mg}$ de $\mathrm{Cu}$ e $5 \mathrm{mg}$ de $\mathrm{Zn}$, omitindo-se quando pertinente, o nutriente referente ao tratamento.

As fontes utilizadas foram reagentes p.a.: $\mathrm{Ca}\left(\mathrm{H}_{2} \mathrm{PO}_{4}\right)_{2} ; \mathrm{KH}_{2} \mathrm{PO}_{4} ; \mathrm{NH}_{4} \mathrm{H}_{2} \mathrm{PO}_{4} ; \mathrm{NaH}_{2} \mathrm{PO}_{4} ; \mathrm{K}_{2} \mathrm{SO}_{4}$; $\mathrm{CaSO}_{4} ; \mathrm{MgSO}_{4} ; \mathrm{Mg}\left(\mathrm{NO}_{3}\right)_{2} ; \mathrm{MgCl}_{2} ; \mathrm{H}_{3} \mathrm{BO}_{3} ; \mathrm{CuCl}_{2} \mathrm{e}$ $\mathrm{ZnCl}_{2}$. As doses das fontes foram calculadas de modo a atender corretamente à adubação básica para cada tratamento. A irrigação foi feita com água destilada a fim de evitar a contaminação e comprometer a influência da adubação.

As avaliações de artrópodes e da percentagem

Rev. Bras. Pl. Med., Botucatu, v.11, n.1, p.18-23, 2009. 
de danos foram semanais por contagem direta, na parte da manhã, em duas folhas apicais, duas medianas e duas basais de cada planta, nas faces adaxial e abaxial. Os dados do experimento foram submetidos à ANVA e ao teste de Scott-Knott, bem como a análise de correlação de Pearson, todos a $5 \%$ de probabilidade.

\section{RESULTADO E DISCUSSÃO}

O maior número de ácaros Tetranychus sp. (Acari: Tetranychidae), na face abaxial dos terços médio e basal das plantas com 90 dias de idade, foi observado nos tratamentos 3, 6, 9 e 13 e os maiores danos nos tratamentos 6 e 13 do que nos demais tratamentos, resultando em inúmeras pontuações cloróticas nas folhas, e conseqüentemente, perda foliar fotossintetizante (Tabelas 1 e 2).

Quanto à cochonilha Insignorthezia insignis (Browne) (Hemiptera: Ortheziidae), a maior ocorrência foi verificada também no tratamento 13 , com maior número na face abaxial do terço apical em plantas com mais de 90 dias de idade (Tabelas 1 e 2).

Os fatos prováveis de maiores danos de Tetranychus sp. nas mudas de alecrim-pimenta dos tratamentos 6 e 13 e de I. insignis no último tratamento foram por estes não conterem $\mathrm{P} \mathrm{e} \mathrm{Mg}$, respectivamente, aliado ao suprimento com N. Plantas com deficiência de $\mathrm{P}$ e de $\mathrm{Mg}$ podem bloquear a síntese protéica resultando no acumulo de aminoácidos livres (Marschner, 1995), provendo melhor alimentação desse artrópode (Chaboussou, 1999; Gallo et al., 2002).

Notaram-se maiores números de pulgões alados Aphis gossypii Glover (Hemiptera: Aphididae), na face abaxial dos terços médio e basal das plantas entre 60 e 90 dias de idade, nos tratamentos 5, 6, 7, 8,11 e 13 , entretanto, colonizou preferencialmente as plantas do tratamento 4 (Tabelas 1 e 2). $O$ ataque intenso de pulgões nas plantas do tratamento 4 resultou em formação de fumagina nas folhas, advindo o ataque de fungos no substrato formado pelo líquido açucarado eliminado pelos pulgões, como resultado da sucção de seiva das plantas.

Observou-se maior ataque da cochonilha Phenacoccus sp. (Hemiptera: Pseudococcidae) nas plantas do tratamento 4 , preferencialmente na face abaxial da parte apical das plantas com mais de 120 dias de idade (Tabelas 1 e 2).

A plausível justificativa da preferência de $A$. gossypiie Phenacoccus sp. pelas plantas de alecrimpimenta do tratamento 4 se deve, provavelmente, ao fato da omissão do elemento $\mathrm{Ca}$, por meio de suplemento ou pelo calcário, aliado ao complemento com N, fatos que o destaca dos demais tratamentos, sendo o Ca participante da síntese de parede celular e lignina (Taiz \& Zeiger, 2004) e indutor da resistência de plantas ao ataque de insetos sugadores (Correa et al., 2005; Gomes et al., 2005).

Foram mais observados adultos de Bemisa tabaci (Genn.) (Hemiptera: Aleyrodidae), na face abaxial dos terços médio e basal de plantas entre 60 e 90 dias de idade, nos tratamentos 5, 7, 8 e 13 e de ninfas, o que indica maior colonização de fato, nestes três últimos tratamentos (Tabelas 1 e 2). As maiores colonizações nas plantas dos tratamentos 7, 8 e 13 provavelmente se devem às deficiências de $\mathrm{K}, \mathrm{S}$ e $\mathrm{Mg}$, respectivamente, aliado à não deficiência de $\mathrm{N}$.

OK faz parte da síntese da RNA polimerase, o S está envolvido diretamente na composição de aminoácidos e o Mg na síntese protéica (Marschner, 1995; Taiz \& Zeiger, 2004). Ou seja, a deficiência desses elementos resulta em acúmulo de aminoácidos livres na seiva (Marschner, 1995). Espécies de insetos sugadores como pulgões, cochonilhas, cigarrinhas, tripes e várias espécies de ácaros fitófagos não são capazes de desdobrar proteínas em aminoácidos para serem utilizados à conveniência própria de cada inseto, por isso eles dependem de aminoácidos livres, existentes na seiva ou no suco celular das plantas (Gallo et al., 2002).

Não se detectou diferença significativa do efeito dos tratamentos sobre a incidência de Frankliniella sp. (Thysanoptera: Thripidae), estando este mais presente na face abaxial das folhas apicais das plantas com mais de 90 dias de idade (Tabela 2). Dentre os artrópodes fitófagos, tripes foi o de menor ocorrência, o que pode explicar a não diferenciação entre os tratamentos.

Observaram-se biorreguladores como o predador Scymnus sp. (Coleoptera: Coccinelidae), predadores de ácaros fitófagos $(r=0,17 ; P=0,0001)$, mosca-branca $(r=0,12 ; P=0,0001)$, cochonilhas $(P>$ $0,05)$ e pulgões $(r=0,22 ; P=0,0001)$; vespa parasitóide de pulgões (Hymenoptera: Braconidae) $(r=0,42 ; P=0,0001)$ e os protocooperantes de sugadores, as formigas (Hymenoptera: Formicidae) $(r=0,11 ; P=0,0001)$, nos tratamentos, nas partes das plantas e períodos de maior ocorrência de suas presas e hospedeiras (Tabelas 1 e 2). Outro inimigo natural observado em plantas de alecrim-pimenta foi aranha Misumenops sp. (Thomisidae), predadora generalista e, neste trabalho, não apresentou correlação significativa com nenhum dos herbívoros citados.

Esses inimigos naturais são importantes agentes reguladores de insetos fitófagos em condições de campo ou de casa-de-vegetação, devendo ser preservados (Gallo et al., 2002; Soglia et al., 2006; Vásquez et al., 2006). Por outro lado, formigas dificultam a ação de inimigos naturais por proteger os sugadores destes quando apresentam protocooperação (Fagundes et al., 2005; Moog et al., 2005).

Rev. Bras. PI. Med., Botucatu, v.11, n.1, p.18-23, 2009. 
TABELA 1. Efeitos dos elementos faltantes sobre percentagem de dano/folha e número de artrópodes/folíolo em Lippia sidoides em Montes Claros-MG, 2006.

\begin{tabular}{|c|c|c|c|c|}
\hline Tratamentos & Tetranychus sp. (dano) & Tetranychus sp. & Aphis gossypiï & Aphis gossyoü (alado) \\
\hline T1 & $2,45 \mathrm{C}$ & $0,64 \mathrm{C}$ & $3,23 \mathrm{C}$ & $0,05 \mathrm{~B}$ \\
\hline T 2 & $6,17 \mathrm{C}$ & $1,61 \mathrm{~B}$ & $4,74 \mathrm{~B}$ & $0,05 \mathrm{~B}$ \\
\hline T 3 & $7,34 \mathrm{~B}$ & $2,49 \mathrm{~A}$ & $7,65 \mathrm{~B}$ & $0,03 \mathrm{~B}$ \\
\hline T 4 & $2,87 \mathrm{C}$ & $0,87 \mathrm{C}$ & $13,55 \mathrm{~A}$ & $0,04 \mathrm{~B}$ \\
\hline T 5 & $4,31 \mathrm{C}$ & $0,68 \mathrm{C}$ & $4,75 \mathrm{C}$ & $0,09 \mathrm{~A}$ \\
\hline T 6 & $10,41 \mathrm{~A}$ & $2,39 \mathrm{~A}$ & $3,61 \mathrm{C}$ & $0,08 \mathrm{~A}$ \\
\hline T 7 & $5,03 \mathrm{C}$ & $1,74 \mathrm{~B}$ & $6,60 \mathrm{~B}$ & $0,09 \mathrm{~A}$ \\
\hline T 8 & $2,93 \mathrm{C}$ & $1,11 \mathrm{C}$ & $6,86 \mathrm{~B}$ & $0,13 \mathrm{~A}$ \\
\hline T 9 & $7,67 \mathrm{~B}$ & $2,28 \mathrm{~A}$ & $6,06 \mathrm{~B}$ & $0,06 \mathrm{~B}$ \\
\hline $\mathrm{T} 10$ & $4,88 \mathrm{C}$ & $1,02 \mathrm{C}$ & $3,82 \mathrm{C}$ & $0,03 \mathrm{~B}$ \\
\hline T 11 & $3,54 \mathrm{C}$ & $0,73 \mathrm{C}$ & $6,38 \mathrm{~B}$ & $0,11 \mathrm{~A}$ \\
\hline Т 12 & $6,13 \mathrm{~B}$ & $1,51 \mathrm{~B}$ & $2,26 \mathrm{C}$ & $0,03 \mathrm{~B}$ \\
\hline \multirow[t]{2}{*}{$\mathrm{T} 13$} & $9,43 \mathrm{~A}$ & $2,36 \mathrm{~A}$ & $2,92 \mathrm{C}$ & $0,10 \mathrm{~A}$ \\
\hline & Insignarthezia insignis & Phenacoccus sp. & B. tabaci (ninfa) & Bemisia tabaci (adulto) \\
\hline T1 & $0,000 \mathrm{~B}$ & $1,12 \mathrm{~B}$ & $0,000 \mathrm{~B}$ & $0,01 \mathrm{~B}$ \\
\hline T 2 & $0,011 \mathrm{~B}$ & $0,82 \mathrm{~B}$ & $0,003 \mathrm{~B}$ & $0,02 \mathrm{~B}$ \\
\hline T 3 & $0,006 \mathrm{~B}$ & $0,51 \mathrm{~B}$ & $0,000 \mathrm{~B}$ & $0,01 \mathrm{~B}$ \\
\hline $\mathrm{T}_{4}$ & $0,006 \mathrm{~B}$ & $3,24 \mathrm{~A}$ & $0,002 \mathrm{~B}$ & $0,01 \mathrm{~B}$ \\
\hline T 5 & $0,002 \mathrm{~B}$ & $0,51 \mathrm{~B}$ & $0,005 \mathrm{~B}$ & $0,03 \mathrm{~A}$ \\
\hline T 6 & $0,051 \mathrm{~B}$ & $0,36 \mathrm{~B}$ & $0,000 \mathrm{~B}$ & $0,02 \mathrm{~B}$ \\
\hline T 7 & $0,003 \mathrm{~B}$ & $0,73 \mathrm{~B}$ & $0,047 \mathrm{~A}$ & $0,06 \mathrm{~A}$ \\
\hline T $B$ & $0,011 \mathrm{~B}$ & $0,71 \mathrm{~B}$ & $0,054 \mathrm{~A}$ & $0,05 \mathrm{~A}$ \\
\hline T 9 & $0,002 \mathrm{~B}$ & $1,92 \mathrm{~B}$ & $0,000 \mathrm{~B}$ & $0,02 \mathrm{~B}$ \\
\hline T 10 & $0,006 \mathrm{~B}$ & $0,41 \mathrm{~B}$ & $0,008 \mathrm{~B}$ & $0,02 \mathrm{~B}$ \\
\hline T 11 & $0,005 \mathrm{~B}$ & $0.14 \mathrm{~B}$ & $0,011 \mathrm{~B}$ & $0,02 \mathrm{~B}$ \\
\hline $\mathrm{T} 12$ & $0,000 \mathrm{~B}$ & $0,47 \mathrm{~B}$ & $0,000 \mathrm{~B}$ & $0,01 \mathrm{~B}$ \\
\hline \multirow[t]{2}{*}{ T 13} & $0,170 \mathrm{~A}$ & $0,34 \mathrm{~B}$ & $0,040 \mathrm{~A}$ & $0,04 \mathrm{~A}$ \\
\hline & Formiga & Scymnus sp. & \multicolumn{2}{|c|}{ Aphis gossypü mumificado } \\
\hline T1 & $0,000 \mathrm{~B}$ & $0,003 \mathrm{~B}$ & \multicolumn{2}{|r|}{$0,06 \mathrm{C}$} \\
\hline T 2 & $0,000 \mathrm{~B}$ & $0,006 \mathrm{~B}$ & \multicolumn{2}{|r|}{$0,38 \mathrm{~A}$} \\
\hline T 3 & $0,000 \mathrm{~B}$ & $0,003 \mathrm{~B}$ & \multicolumn{2}{|r|}{$0,54 \mathrm{~A}$} \\
\hline T 4 & $0,000 \mathrm{~B}$ & $0,013 \mathrm{~A}$ & \multicolumn{2}{|r|}{$0,53 \mathrm{~A}$} \\
\hline T 5 & $0,000 \mathrm{~B}$ & $0,002 \mathrm{~B}$ & \multicolumn{2}{|r|}{$0,24 \mathrm{~B}$} \\
\hline T 6 & $0,006 \mathrm{~B}$ & $0,000 \mathrm{~B}$ & \multicolumn{2}{|r|}{$0,13 \mathrm{C}$} \\
\hline T 7 & $0,006 \mathrm{~B}$ & $0,010 \mathrm{~A}$ & \multicolumn{2}{|r|}{$0,61 \mathrm{~A}$} \\
\hline T 8 & $0,013 \mathrm{~A}$ & $0,006 \mathrm{~B}$ & \multicolumn{2}{|r|}{$0,21 \mathrm{C}$} \\
\hline T 9 & $0,019 \mathrm{~A}$ & $0,016 \mathrm{~A}$ & \multicolumn{2}{|r|}{$0,27 \mathrm{~B}$} \\
\hline T 10 & $0,010 \mathrm{~A}$ & $0,005 \mathrm{~B}$ & \multicolumn{2}{|r|}{$0,30 \mathrm{~B}$} \\
\hline T 11 & $0,000 \mathrm{~B}$ & $0,000 \mathrm{~B}$ & \multicolumn{2}{|r|}{$0,35 \mathrm{~B}$} \\
\hline $\mathrm{T} 12$ & $0,006 \mathrm{~B}$ & $0,013 \mathrm{~A}$ & \multicolumn{2}{|r|}{$0,19 \mathrm{C}$} \\
\hline T 13 & $0,000 \mathrm{~B}$ & $0,026 \mathrm{~A}$ & \multicolumn{2}{|r|}{$0,35 \mathrm{~B}$} \\
\hline
\end{tabular}

$\mathrm{T} 1$ = testemunha (sem adubação e sem calagem), T2 = completo $1(\mathrm{~N}, \mathrm{P}, \mathrm{K}, \mathrm{S}, \mathrm{B}, \mathrm{Cu}, \mathrm{Zn}+\mathrm{calagem}$ ), T3 = completo 2 (completo 1 sem calagem mais $\mathrm{Ca}$ e Mg como sulfato), $\mathrm{T} 4=$ completo 1 sem calagem, $\mathrm{T} 5=$ completo $1 \mathrm{sem} \mathrm{N}, \mathrm{T} 6=$ completo $1 \mathrm{sem} \mathrm{P}, \mathrm{T} 7$ = completo 1 sem $\mathrm{K}, \mathrm{T} 8=$ completo $1 \mathrm{sem} \mathrm{S}, \mathrm{T} 9=$ completo $1 \mathrm{sem} \mathrm{B}, \mathrm{T} 10=$ completo $1 \mathrm{sem} \mathrm{Cu}, \mathrm{T} 11=$ completo $1 \mathrm{sem} \mathrm{Zn}, \mathrm{T} 12=$ completo 1 sem $\mathrm{Ca}$ e $\mathrm{T} 13=$ completo 2 sem Mg. As médias seguidas pela mesma letra maiúscula na coluna não diferem, entre si, pelo teste de média de Scott-Knott a $5 \%$ de significância. 
TABELA 2. Efeito de dossel de plantas, face foliar e idade de plantas (dias) sobre a percentagem de dano/folha e de artrópodes/face foliar em Lippia sidoides em Montes Claros-MG. 2006.

\begin{tabular}{|c|c|c|c|c|}
\hline \multirow[t]{2}{*}{ Danos ou artrópodes } & \multicolumn{4}{|c|}{ Efeito de dossel de plantas } \\
\hline & Apical & Médio & & Basal \\
\hline Tetranychus sp. (dano) & $0,75 \mathrm{~B}$ & $7,15 \mathrm{~A}$ & & $8,99 \mathrm{~A}$ \\
\hline Tetranychus sp. & $0,74 \mathrm{C}$ & $2,25 \mathrm{~A}$ & & $1,49 \mathrm{~B}$ \\
\hline Aphis gossypï & $4,12 \mathrm{~B}$ & $6,76 \mathrm{~A}$ & & $5,85 \mathrm{~A}$ \\
\hline Insignorthezia insignis & $0,13 \mathrm{~A}$ & $0,03 \mathrm{~B}$ & & $0,03 \mathrm{~B}$ \\
\hline Phenacoccus sp & $0,99 \mathrm{~A}$ & $0,84 \mathrm{~B}$ & & $0,78 \mathrm{~B}$ \\
\hline Bemisia tabaci (ninfa) & $0,00 \mathrm{~B}$ & $0,02 \mathrm{~A}$ & & $0,02 \mathrm{~A}$ \\
\hline Frankiniells sp. & $0,000 \mathrm{~B}$ & $0,003 \mathrm{~B}$ & & $0,017 \mathrm{~A}$ \\
\hline Scymnus sp. (larva) & $0,01 \mathrm{~B}$ & $0,04 \mathrm{~A}$ & & $0,03 \mathrm{~A}$ \\
\hline Aphis gossypï (mumificado) & $0,06 \mathrm{C}$ & $0,29 \mathrm{~B}$ & & $0,62 \mathrm{~A}$ \\
\hline Aranhas & $0,012 \mathrm{~A}$ & $0,006 \mathrm{~B}$ & & $0,004 \mathrm{~B}$ \\
\hline \multirow[t]{2}{*}{ Danos ou artrópodes } & \multicolumn{4}{|c|}{ Face foliar } \\
\hline & \multicolumn{2}{|c|}{ Adaxial } & \multicolumn{2}{|c|}{ Abaxial } \\
\hline Tetranychus sp. & \multicolumn{2}{|c|}{$0,21 \mathrm{~B}$} & \multicolumn{2}{|c|}{$2,78 \mathrm{~A}$} \\
\hline Aphis gossypï & \multicolumn{2}{|c|}{$1,00 \mathrm{~B}$} & \multicolumn{2}{|c|}{$10,15 \mathrm{~A}$} \\
\hline Aphis gossypö (alado) & \multicolumn{2}{|c|}{$0,01 \mathrm{~B}$} & \multicolumn{2}{|c|}{$0,13 \mathrm{~A}$} \\
\hline Insignorthezia insignis & \multicolumn{2}{|c|}{$0,04 \mathrm{~B}$} & \multicolumn{2}{|c|}{$0,09 \mathrm{~A}$} \\
\hline Phenacoccus sp & \multicolumn{2}{|c|}{0,27 B } & \multicolumn{2}{|c|}{$1,47 \mathrm{~A}$} \\
\hline Bemisia tabaci (Adulto) & \multicolumn{2}{|c|}{$0,01 \mathrm{~B}$} & \multicolumn{2}{|c|}{$0,05 \mathrm{~A}$} \\
\hline B. tabaci (ninfa) & \multicolumn{2}{|c|}{$0,00 \mathrm{~B}$} & \multicolumn{2}{|c|}{$0,03 \mathrm{~A}$} \\
\hline Frankliniella sp. & \multicolumn{2}{|c|}{$0,00 \mathrm{~B}$} & \multicolumn{2}{|c|}{$0,01 \mathrm{~A}$} \\
\hline Scymnus sp. (larva) & \multicolumn{2}{|c|}{$0,01 \mathrm{~B}$} & \multicolumn{2}{|c|}{$0,05 \mathrm{~A}$} \\
\hline Scymnus sp. (adulto) & \multicolumn{2}{|c|}{$0,00 \mathrm{~B}$} & & $01 \mathrm{~A}$ \\
\hline A. gassypï mumificado & & & & $62 \mathrm{~A}$ \\
\hline Braconidae & & & & $02 \mathrm{~A}$ \\
\hline Aranhas & & & & $01 \mathrm{~A}$ \\
\hline Danos ou artrópodes & & lade de pla & ntas & \\
\hline & 30 dias & 60 dias & go dias & 120 dias \\
\hline Tetranychus sp. (dano) & $0,22 \mathrm{D}$ & $2,04 \mathrm{C}$ & $13,29 \mathrm{~A}$ & $10,97 \mathrm{~B}$ \\
\hline Tetranychus sp. & $0,04 \mathrm{C}$ & $0,92 \mathrm{~B}$ & $3,88 \mathrm{~A}$ & $0,06 \mathrm{C}$ \\
\hline Aphis gossypï & $1,06 \mathrm{~B}$ & $7,95 \mathrm{~A}$ & $9,00 \mathrm{~A}$ & $0,44 \mathrm{~B}$ \\
\hline A. gossypir (alado) & $0,03 \mathrm{~B}$ & $0,19 \mathrm{~A}$ & $0,04 \mathrm{~B}$ & $0,00 \mathrm{C}$ \\
\hline Insignorthezia insignis & $0,02 \mathrm{~B}$ & $0,04 \mathrm{~B}$ & $0,13 \mathrm{~A}$ & $0,15 \mathrm{~A}$ \\
\hline Phenacoccus sp. & $0,04 \mathrm{C}$ & $0,15 \mathrm{C}$ & $1,68 \mathrm{~B}$ & $3,84 \mathrm{~A}$ \\
\hline Bemisig tabaci (adulto) & $0,004 \mathrm{C}$ & $0,022 \mathrm{~B}$ & $0,061 \mathrm{~A}$ & $0,006 \mathrm{C}$ \\
\hline B. tabaci (ninfa) & $0,00 \mathrm{~B}$ & $0,03 \mathrm{~A}$ & $0,02 \mathrm{~A}$ & $0,00 \mathrm{~B}$ \\
\hline Frankliniella sp. & $0,00 \mathrm{~B}$ & $0,00 \mathrm{~B}$ & $0,02 \mathrm{~A}$ & $0,01 \mathrm{~A}$ \\
\hline Scymnus sp. (larva) & $0,00 \mathrm{~B}$ & $0,01 \mathrm{~B}$ & $0,08 \mathrm{~A}$ & $0,01 \mathrm{~B}$ \\
\hline Scymnus sp. (adulto) & $0,000 \mathrm{~B}$ & $0,002 \mathrm{~B}$ & $0,022 \mathrm{~A}$ & $0,003 \mathrm{~B}$ \\
\hline Pulgão mumificado & $0,01 \mathrm{D}$ & $0,40 \mathrm{~B}$ & $0,60 \mathrm{~A}$ & $0,14 \mathrm{C}$ \\
\hline Braconidae & $0,00 \mathrm{C}$ & $0,02 \mathrm{~A}$ & $0,01 \mathrm{~B}$ & $0,00 \mathrm{C}$ \\
\hline Aranha & $0,003 \mathrm{C}$ & $0,003 \mathrm{C}$ & $0,012 \mathrm{~B}$ & $0.022 \mathrm{~A}$ \\
\hline Formiga & $0,000 \mathrm{C}$ & $0,002 \mathrm{C}$ & $0,007 \mathrm{~B}$ & $0,022 \mathrm{~A}$ \\
\hline
\end{tabular}

As médias seguidas pela mesma letra maiúscula na linha não diferem, entre si, pelo teste de média de Scott-Knott a 5\% de significância. 
Em resumo, os tratamentos $\mathrm{T} 1, \mathrm{~T} 5$ e $\mathrm{T} 10$ foram os menos atacados por artrópodes fitófagos. Dirigir a pulverização, quando necessária, sempre para a face inferior da folha, local preferencial de ataque dos artrópodes.

\section{AGRADECIMENTO}

Ao Dr. Victor Pirovani e Prof. Paulo Sérgio Fiuza Ferreira (Universidade Federal de Viçosa) e Profa Ana Lúcia Benfatti Gonzalez Peronti (Universidade Federal de São Carlos) pelas identificações de cochonilhas e ao financiamento do Banco do Nordeste.

\section{REFERÊNCIA}

BARROSO, D.G. et al. Diagnóstico de deficiência de macronutrientes em mudas de teca. Revista Árvore, v.29, n.5, p.671-9, 2005.

CARVALHO, A.F.U. et al. Larvicidal activity of the essential oil from Lippia sidoides cham. against Aedes aegypti Linn. Memorial Instituto Oswaldo Cruz, v.98, n.4, p.56971, 2003.

CHABOUSSOU, F. Plantas doentes pelo uso de agrotóxicos: a teoria da trofobiose. 2.ed. Porto Alegre: L\&PM, 1999. 272p.

CORREA, R.S.B. et al. Silicon and acibenzolar-S-methyl as resistance inducers in cucumber, against the whitefly Bemisia tabaci (Gennadius) (Hemiptera: Aleyrodidae) biotype B. Neotropical Entomology, v.34, n.3, p.429-33, 2005.

COSTA, S.M. et al. Chemical constituents from Lippia sidoides and cytotoxic activity. Journal of Natural Products, v.64, n.6, p.792-5, 2001.

EMPRESABRASILEIRADE PESQUISAAGROPECUÁRIACentro Nacional de Pesquisa da Amazônia Oriental, (EMBRAPA-CPATU. Documentos). Belém: EMBRAPA/ CPATU, 1997. 148p.

EMPRESABRASILEIRADE PESQUISAAGROPECUÁRIACentro Nacional de Pesquisa de Solos. Sistema Brasileiro de Classificação de Solos. Brasília: Embrapa Produção de Informação; Rio de Janeiro: Embrapa Solos, 1999. 412p.

FAGUNDES, M.; NEVES, F.S.; FERNANDES, G.W. Direct and indirect interactions involving ants, insects herbivores, parasitoids and the host plant Baccharis dracunculifolia (Asteraceae). Ecological Entomology, v.30, p.28-35, 2005.
FERNANDES, L.A. et al. Nutrição mineral de plantas de maxixe-do-reino. Pesquisa Agropecuária Brasileira, v.40, n.7, p.719-22, 2005.

GALLO, D. et al. Entomologia agrícola. Piracicaba: FEALQ, 2002. 920p.

GIRÃO, V.C. et al. A clinical trial of the effect of a mouthrinse prepared with Lippia sidoides Cham essential oil in dogs with mild gingival disease. Preventive Veterinary Medicine, v.59, n.1-2, p.95-102, 2003.

GOMES, F.B. et al. Resistance induction in wheat plants by silicon and aphids. Scientia Agricola, v.62, n.6, p.54751, 2005.

LORENZI, H.; MATOS, F.J.A. Plantas medicinais no Brasil: nativas e exóticas cultivadas. Nova Odessa: Instituto Plantarum, 2002. 530p.

MAPELI, N.C. et al. Produção de biomassa e de óleo essencial dos capítulos florais da camomila em função de nitrogênio e fósforo. Horticultura Brasileira, v.23, n.1, p.32-7, 2005.

MARSCHNER, H. Mineral nutrition of higher plants. London: Academic Press, 1995. 889p.

MARTINS, E.R. et al. Plantas medicinais. Viçosa: UFV, 1994. 220p.

MOOG, J. et al. The triple alliance: how a plant-ant, living in an ant-plant, acquires the third partner, a scale insect. Insectes Sociaux, v.52, n.2, p.169-76, 2005.

RIBEIRO, A.C.; GUIMARÃES, P.T.G.; ALVAREZ, V.H. Comissão de fertilidade do solo do Estado de Minas Gerais. Recomendações para o uso de corretivos e fertilizantes em Minas Gerais - 5. Aproximação. Viçosa: UFV, 1999. 359p.

SIMÕES, C.M.O. et al. Farmacognosia: da planta ao medicamento. Porto Alegre: Ed. Universitária, 2000. $821 \mathrm{p}$.

SOGLIA, M.C.M. et al. Desenvolvimento e parasitismo de Lysiphlebus testaceipes (Cresson) e Aphidius colemani Viereck (Hymenoptera: Braconidae) em Aphis gossypii Glover (Hemiptera: Aphididae) em duas cultivares de crisântemo. Neotropical Entomology, v.35, n.3, p.364-70, 2006.

TAIZ, L.; ZEIGER, E. Fisiologia vegetal. Porto Alegre: Artmed, 2004. 719p.

VÁSQUEZ, G.M.; ORR, D.B.; BAKER, J.R. Efficacy Assessment of Aphidius colemani (Hymenoptera: Braconidae) for Suppression of Aphis gossypii (Homoptera: Aphididae) in Greenhouse-Grown Chrysanthemum. Journal of Economic Entomology, v.99, p.1104-11, 2006.

VENTURIN, N. et al. Adubação mineral do angicoamarelo [Peltophorum dubium (Spreng.) Taub.]. Pesquisa Agropecuária Brasileira, v.34, n.3, p.441-8, 1999. 